\title{
Isolated Leptomeningeal Progression in a Patient with NTRK Fusion+ Uterine Sarcoma: A Case Report
}

\author{
Tyler Lanman ${ }^{a} \quad$ Melanie Hayden Gephart ${ }^{b} \quad$ Nam Buic $^{c} \quad$ Angus Toland ${ }^{d}$ \\ Seema Nagpal \\ aDepartment of Neurology, Stanford University, Stanford, CA, USA; ${ }^{b}$ Department of \\ Neurosurgery, Stanford University, Stanford, CA, USA; 'Department of Medicine, Oncology, \\ Stanford University, Stanford, CA, USA; dDepartment of Pathology, Stanford University, \\ Stanford, CA, USA; 'Department of Neurology, Neuro-Oncology, Stanford University, \\ Stanford, CA, USA
}

\author{
Keywords \\ Neurotrophic tropomyosin receptor kinase - Leptomeningeal disease - Brain metastases . \\ Larotrectinib · Uterine sarcoma
}

\begin{abstract}
While neurotrophic tropomyosin receptor kinase (NTRK) fusions represent rare oncogenic drivers ( $<1 \%$ of solid cancers), the recent approval of NTRK inhibitors (larotrectinib and entrectinib) led to dramatic responses in patients with NTRK fusion+ tumors. Both drugs have phase I data, demonstrating efficacy in the central nervous system (CNS), including both primary brain tumors and brain metastases. We present a 29-year-old woman who was diagnosed with NTRK3-SPECC1L fusion+ undifferentiated uterine sarcoma and underwent resection, chemotherapy, and radiotherapy. Two years later, lung metastases were discovered. She was started on larotrectinib with complete response. She remained stable on larotrectinib until she presented with altered mental status and seizures. MRI demonstrated leptomeningeal enhancement, but because leptomeningeal progression from sarcoma is exceedingly rare and her symptoms improved dramatically with antiepileptics, these findings were initially attributed to seizures. After 2 unrevealing lumbar punctures and stable systemic imaging, a brain biopsy demonstrated metastatic sarcoma, still showing NTRK positivity. She underwent whole brain radiotherapy and was switched to entrectinib, but had clinical progression 1 month later and transitioned to hospice. This case demonstrates the efficacy of NTRK inhibitors in rare and aggressive cancer but highlights that these patients can develop isolated CNS progression even in the setting of CNS-penetrant drugs. CNS progression can occur if there is incom-
\end{abstract}


plete CNS drug penetration, discordance in molecular profiles between CNS and systemic disease, or acquired NTRK inhibitor resistance. In this case, CNS disease maintained the NTRK fusion status, but either inadequate CNS penetration or development of a resistance gene may explain the isolated CNS progression.

(C) 2021 The Author(s).

Published by S. Karger AG, Basel

\section{Introduction}

Neurotrophic tropomyosin receptor kinase (NTRK) is a family of genes (NTRK1, NTRK2, and NTRK3) that, respectively, encode proteins TrkA, B, and C, which play roles in cell signaling. Fusion events involving one of these genes may result in constitutive tyrosine kinase activity, leading to the downstream activation of several pathways (RAS/RAF/MAPK, $\mathrm{PI} 3 \mathrm{~K} / \mathrm{Akt} / \mathrm{mTOR}$, and PLC/PKC) involved in cellular growth and proliferation [1]. Through this mechanism, NTRK-fusion proteins act as primary oncogenic drivers. NTRK was first implicated as an oncogene in colorectal cancer in the 1980s but has since been described in a variety of tumor types including nonsmall-cell lung carcinomas, salivary gland carcinomas, thyroid carcinomas, and gliomas [2]. With the advent of targeted therapy in oncology, an emphasis was placed on developing tissue-agnostic treatment (meaning a cancer medication that targets a driver mutation rather than the cancer's subtype or primary location) for NTRK. Larotrectinib is a tyrosine kinase inhibitor that blocks the ATP-binding domain of Trk, demonstrating considerable selectivity and potency against all 3 Trk proteins [3]. In a pooled analysis of clinical trials, larotrectinib was found to have a $79 \%$ overall response rate with minimal occurrence of adverse events [4]. In 2018, larotrectinib became the second tissue-agnostic cancer drug to be approved by the FDA and the first targeted therapy for NTRK cancers.

Uterine sarcomas represent a heterogeneous group of rare tumors, accounting for $<3 \%$ of all female genital tract malignancies [5]. Molecular sequencing performed in sarcoma samples found underlying NTRK fusions in $0.68 \%$ of all cases [6]. Furthermore, sarcoma only rarely metastasizes to the brain, with a large prospective trial finding an incidence rate of $<1 \%$ [7]. Here, we report the first described case of a patient diagnosed with uterine sarcoma with NTRK rearrangement who developed isolated leptomeningeal progression while on an NTRK inhibitor.

\section{Case Presentation}

In 2015, a 29-year-old woman developed heavy menstrual bleeding with clinical exam and imaging, demonstrating a $15 \times 10-\mathrm{cm}$ mass involving the cervix. Biopsy and subsequent hysterectomy demonstrated an intermediate grade sarcoma with no definite immunophenotypic pattern of differentiation. Additional molecular testing demonstrated NTRK3SPECC1L chimeric rearrangement. She then underwent 5 cycles of adriamycin/ifosfamide with adjuvant radiation (45 Gy in 25 fractions). In 2016, she was found to have lung metastases and underwent 2 cycles of gemcitabine/docetaxel. After continued disease progression, in 2017, she was enrolled in the LOXO-TRK-15002 phase 1 clinical trial, receiving larotrectinib $100 \mathrm{mg}$ twice daily. This led to an impressive stabilization of her disease and dramatic improvement in lung metastases without significant side effects. She remained on this medication after the trial ended and showed no radiographic or clinical progression for 3 years.

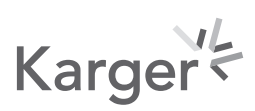



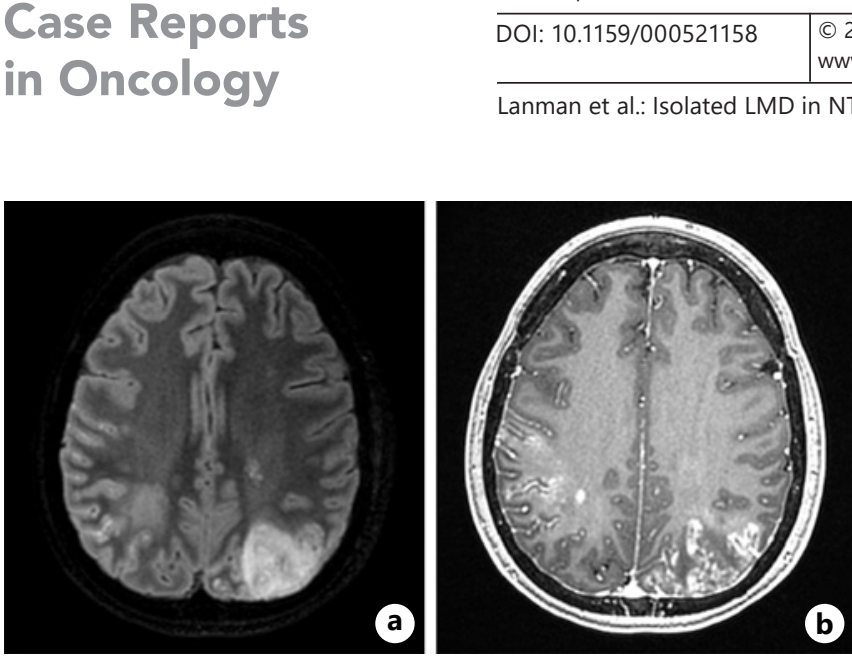

Fig. 1. MRI brain. MRI of the brain with and without contrast showing T2/FLAIR sequence demonstrating a large focal area of T2 signal abnormality in the left occipital region (a), axial T1 BRAVO with contrast sequence demonstrating patchy areas of subcortical and leptomeningeal enhancement (b), Tmax sequence demonstrating prolonged Tmax on color maps within the posterior/superior parietal lobes bilaterally (c), right more than left, locations that correspond with areas of signal abnormality described above.

In 2020, she developed increasing confusion at home over the course of a week. After being found down and incoherent, she was brought to a local hospital and was found to be in status epilepticus. She required levetiracetam, lacosamide, and lamotrigine for seizure stabilization. Her MRI demonstrated multifocal patchy areas of T2 hyperintensities with posterior predominance (Fig. 1). A low-volume lumbar puncture during this admission revealed $\mathrm{WBC}<1, \mathrm{RBC} 31$, glucose 50 , protein 66 , and no atypical cells. Her seizures resolved, her mental status slowly improved, and she was discharged.

She then presented to our neuro-oncology clinic, appearing generally well from a neurological perspective, with mild difficulty giving a linear history (which improved with repeat visits) and otherwise nonfocal neurological exam. At our institution, she underwent a repeat lumbar puncture, which revealed a similar profile to the first and similarly had unremarkable cytology. Repeat brain MRI was stable compared to prior. The specific etiology of her intracranial pathology remained unclear. Leptomeningeal disease (LMD) was considered but felt to be less likely, given the rarity of LMD in sarcoma, especially in the context of stable systemic disease, clinical improvement with antiepileptic therapy, and negative cerebral spinal fluid (CSF) cytology on 2 occasions. The differential for her radiographic findings included MRI changes due to prolonged status epilepticus, multifocal ischemia, and infectious/inflammatory meningoencephalitis (although the CSF profile was bland and infectious panels negative, making this etiology less likely). Since larotrectinib had only been recently approved, side effects from this medication, such as posterior reversible encephalopathy syndrome, were also considered. However, to our knowledge, there are no reported cases of posterior reversible encephalopathy syndrome associated with larotrectinib.

Given her initial clinical improvement, no treatment changes were made. Unfortunately, over the course of 6-8 weeks, confusion became a much more prominent problem. She was reluctant to undergo more invasive diagnostic workup, but after hospital admission for confusion, she and her family agreed to a brain/meningeal biopsy which revealed metastatic sarcoma to the leptomeninges (Fig. 2). With the diagnosis confirmed, she underwent a course of whole brain radiation therapy (30 Gy in 10 fractions). After multidisciplinary discussion, she was switched from larotrectinib to entrectinib. Next-generation sequencing performed on the meningeal tissue did not identify any known resistance mutations (i.e., NTRK3p.G696A, BRAFv600e, or KRASp.G12D) or gene amplifications associated with drug resistance. A month after starting entrectinib, she began experiencing increasing lethargy and confusion, and was found to have further progression of LMD. She ultimately transitioned to hospice. 


\section{Case Reports in Oncology}

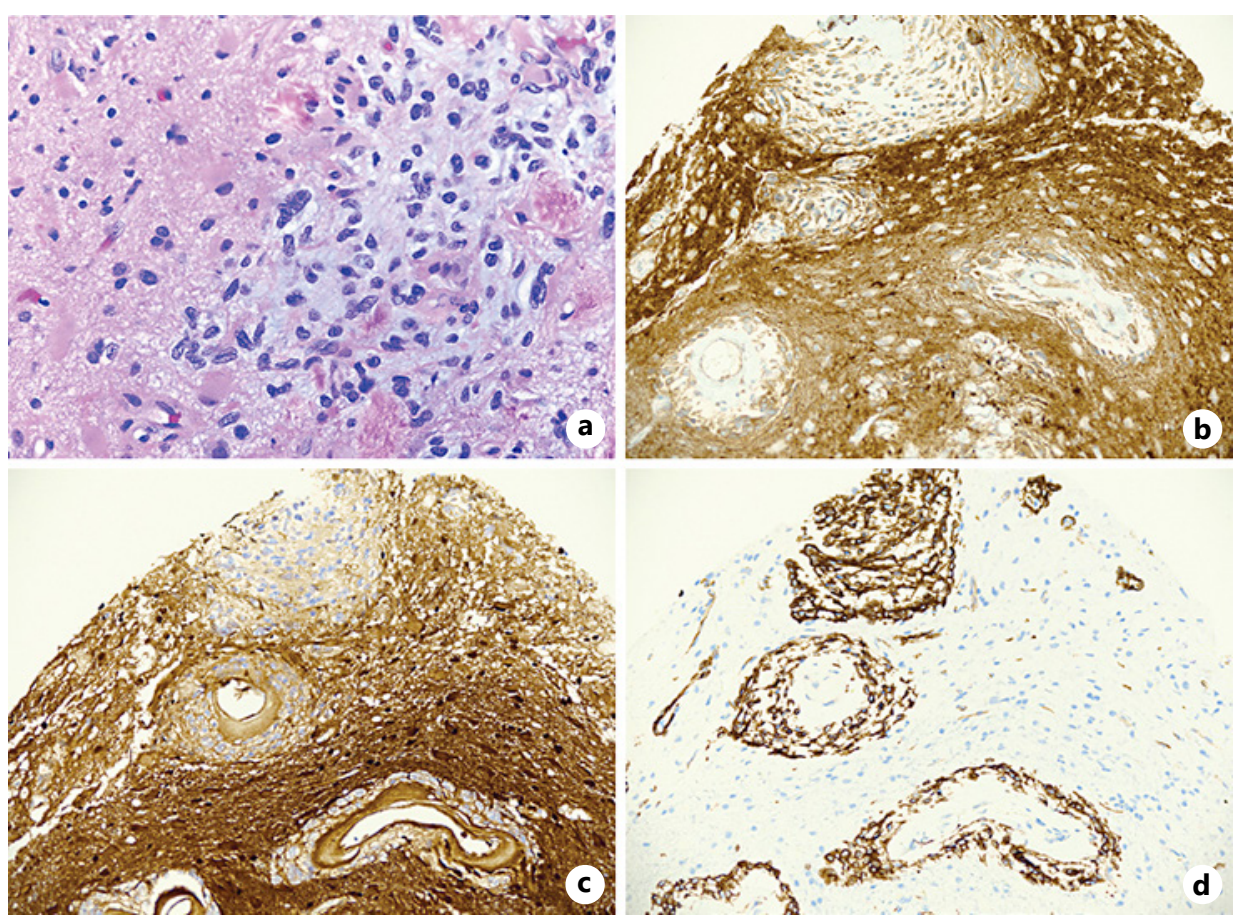

Fig. 2. Tissue pathology from brain/meningeal biopsy showing H\&E stain demonstrating tissue histology (a), positive PanTRK stain indicating that the tissue expresses Trk (b), S100 cytoplasmic immunoreactivity (c), CD34 immunoreactivity (d). H\&E, hematoxylin and eosin.

\section{Discussion}

This patient was one of the first to receive larotrectinib therapeutically via the LOXOTRK-15002 phase I trial. Despite chemotherapy and radiotherapy, the patient had rapidly progressing disease with lung metastases. Considering the poor prognosis of undifferentiated uterine sarcoma (shown to have a progression-free survival of 7.9 months and overall survival of 26.8 months [8]), the initiation of larotrectinib resulted in an impressive and sustained response for 3 years.

From a clinical perspective, this case represents a diagnostic dilemma. The patient initially presented to our clinic after an episode of status epilepticus with unusual MRI findings and a bland CSF profile without tumor cells. While metastatic progression was considered, the negative workup and initial improvement in symptoms without intervention made it less likely. However, with subsequent worsening confusion and recurrent seizures captured on EEG, we felt it was reasonable to consider a meningeal biopsy, which ultimately confirmed leptomeningeal spread of uterine sarcoma. This case underscores the limitations of diagnosing LMD with CSF analysis. CSF cytology has modest sensitivity of $71 \%$ and $86 \%$ for initial and second lumbar puncture, respectively [9]. Higher volumes can increase diagnostic yield, but this was limited in the current patient due to a Chiari malformation. Using tests for CSF circulating tumor cells is also felt to increase the sensitivity of lumbar puncture [10], but our lab does not regularly perform these in-house, and at the time, commercial testing was not available. Additionally, it is not clear that sarcoma cells would be well captured by current commercial testing. Specifically, Veridex uses epithelial cell adhesion molecule, but not all sarcomas express this protein. Biocept is not CLIA approved for sarcoma. Next-generation sequencing panels for cell-free DNA may also be useful in increasing the diagnostic sensitivity 
of lumbar puncture, but fusions can be particularly difficult to detect with the currently available techniques [11].

Metastatic disease to the central nervous system (CNS) despite the presence of an NTRK inhibitor can result from several different mechanisms. InadequateCNS penetration for targeted molecules often contributes to inadequate CNS response in brain metastases/LMD [12]. To overcome this possible mechanism, our team switched to entrectinib, as there is some in vivo/in vitro data to support higher CNS exposure with this NTRK inhibitor [13]. The lack of disease response even after the switch may imply that the CNS penetration between these 2 first-generation NTRK inhibitors is not clinically significant (unless this switch was made too late in the progression to take effect). However, other mechanisms may have played a larger role. Brain metastases can often gain additional mutations, distinct from the somatic primary [14]. However, it would be theoretically feasible but quite unusual to lose an initial driver mutation (in this case, immunohistochemistry of the leptomeningeal tumor showed expression of PanTRK, arguing against loss of NTRK expression). Resistance to targeted therapy has also been documented in NTRK fusion cancers. In 1 study, primary resistance to NTRK inhibition was demonstrated in $11 \%$ of patients, and acquired resistance was found in 18\% [15]. In our patient, next-generation sequencing of the leptomeningeal metastases was unrevealing for a known resistance gene or amplification although it is possible that disease progression was due to a yet-unidentified resistance mechanism. Several second-generation NTRK inhibitors are currently under investigation that preliminarily demonstrate efficacy in NTRK fusion cancers with resistance mutations. In the current case, pursuing compassionate use for selitrectinib was considered, but the patient had opted for hospice.

The main limitation of this case report is its sample size which limits the ability to draw definitive conclusions. However, given the rarity of undifferentiated uterine sarcomas, NTRK fusion cancers, and sarcoma progression to the CNS, this unique presentation provides valuable insight for future clinical management and therapeutic development in this disease entity.

\section{Conclusion}

This case report illustrates several important learning points: a presentation of a rare tumor type with a unique presentation, a demonstration of a complicated neuro-oncological workup, and insight into isolated CNS progression while on a recently approved medication.

\section{Acknowledgments}

We acknowledge and thank the patient and her family for permitting us to share her story. We acknowledge and thank the patient's entire medical team for their care for the patient.

\section{Statement of Ethics}

This case report meets the criteria for IRB exemption as per the Research Compliance Committee, Stanford University. Written informed consent was obtained from the patient's next-of-kin (since patient is deceased) for publication of this case report and any accompanying images. A copy of the written consent is available for review by the editor of this journal.

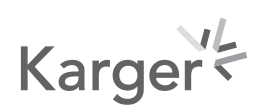




\section{Conflict of Interest Statement}

All authors have no conflicts of interest to declare.

\section{Funding Sources}

This research was not supported by any funding.

\section{Author Contributions}

T.L. and S.M. conceived this case report, obtained consent, and performed chart review. A.T. performed pathological review. All authors significantly contributed to, read, and approved the final manuscript.

\section{Data Availability Statement}

All data generated or analyzed during this study are included in this article. Further inquiries can be directed to the corresponding author.

\section{References}

1 Vaishnavi A, Le AT, Doebele RC. TRKing down an old oncogene in a new era of targeted therapy. Cancer Discov. 2015;5(1):25-34.

2 Amatu A, Sartore-Bianchi A, Siena S. NTRK gene fusions as novel targets of cancer therapy across multiple tumour types. ESMO Open. 2016;1(2):e000023.

3 Ricciuti B, Genova C, Crinò L, Libra M, Leonardi GC. Antitumor activity of larotrectinib in tumors harboring NTRK gene fusions: a short review on the current evidence. Onco Targets Ther. 2019;12:3171.

4 Hong DS, DuBois SG, Kummar S, Farago AF, Albert CM, Rohrberg KS, et al. Larotrectinib in patients with TRK fusionpositive solid tumours: a pooled analysis of three phase 1/2 clinical trials. Lancet Oncol. 2020;21(4):531-40.

5 Livi L, Paiar F, Shah N, Blake P, Villanucci A, Amunni G, et al. Uterine sarcoma: twenty-seven years of experience. Int J Radiat Oncol Biol Phys. 2003;57(5):1366-73.

6 Solomon JP, Linkov I, Rosado A, Mullaney K, Rosen EY, Frosina D, et al. NTRK fusion detection across multiple assays and 33,997 cases: diagnostic implications and pitfalls. Modern Pathol. 2020;33(1):38-46.

7 Espat NJ, Bilsky M, Lewis JJ, Leung D, Brennan MF. Soft tissue sarcoma brain metastases: prevalence in a cohort of 3829 patients. Cancer. 2002;94(10):2706-11.

8 Tanner EJ, Garg K, Leitao MM Jr, Soslow RA, Hensley ML. High grade undifferentiated uterine sarcoma: surgery, treatment, and survival outcomes. Gynecol Oncol. 2012;127(1):27-31.

9 Glantz MJ, Cole BF, Glantz LK, Cobb J, Mills P, Lekos A, et al. Cerebrospinal fluid cytology in patients with cancer: minimizing false-negative results. Cancer. 1998;82(4):733-9.

10 Wooster M, McGuinness JE, Fenn KM, Singh VM, Franks LE, Lee S, et al. Diagnosis of leptomeningeal metastasis through identification of circulating tumor cells in cerebrospinal fluid using the TargetSelector ${ }^{\mathrm{TM}}$ assay. 2021.

11 Boire A, Brandsma D, Brastianos PK, Le Rhun E, Ahluwalia M, Junck L, et al. Liquid biopsy in central nervous system metastases: a RANO review and proposals for clinical applications. Neuro-oncology. 2019;21(5):571-84.

12 Nayar G, Ejikeme T, Chongsathidkiet P, Elsamadicy AA, Blackwell KL, Clarke JM, et al. Leptomeningeal disease: current diagnostic and therapeutic strategies. Oncotarget. 2017;8(42):73312.

13 Fischer H, Ullah M, de la Cruz CC, Hunsaker T, Senn C, Wirz T, et al. Entrectinib, a TRK/ROS1 inhibitor with anti-CNS tumor activity: differentiation from other inhibitors in its class due to weak interaction with P-glycoprotein. Neuro-oncology. 2020;22(6):819-29.

14 Brastianos PK, Carter SL, Santagata S, Cahill DP, Taylor-Weiner A, Jones RT, et al. Genomic characterization of brain metastases reveals branched evolution and potential therapeutic targets. Cancer Discov. 2015;5(11):1164-77.

15 Drilon A, Laetsch TW, Kummar S, DuBois SG, Lassen UN, Demetri GD, et al. Efficacy of larotrectinib in TRK fusion-positive cancers in adults and children. N Engl J Med. 2018;378(8):731-9.

\section{Karger'}

